\title{
Achados histopatológicos em retinoblastoma
}

\author{
Histopathologic findings in retinoblastoma
}

\author{
João Pessoa de Souza Filho ${ }^{1}$ \\ Maria Cristina Martins ${ }^{2}$ \\ Virgínia Laura Torres ${ }^{3}$ \\ Ana Beatriz Toledo Dias ${ }^{4}$ \\ Márcia Serva Lowen ${ }^{5}$ \\ Luciana Afonso Pires ${ }^{6}$ \\ Clélia Maria Erwenne ${ }^{z}$
}

\footnotetext{
${ }^{1}$ Médico Oftalmologista - Pós graduando nível Doutorado - Departamento de Oftalmologia Universidade Federal de São Paulo (UNIFESP). São Paulo (SP).

${ }^{2}$ Chefe do setor de Anatomia Patológica Ocular - Departamento de Oftalmologia da UNIFESP. São Paulo (SP).

${ }^{3}$ Médica Oftalmologista - Estagiária do setor de Anatomia Patológica Ocular - Departamento de Oftalmologia da UNIFESP. São Paulo (SP).

${ }^{4}$ Tecnóloga dos setores de Anatomia Patológica e Farmacologia Ocular - Departamento de Oftalmologia da UNIFESP. São Paulo (SP).

${ }^{5}$ Médica Patologista - Departamento de Anatomia Patológica da UNIFESP. São Paulo (SP).

${ }^{6}$ Médica Oftalmologista - Estagiária do setor de Anatomia Patológica Ocular - Departamento de Oftalmologia da UNIFESP. São Paulo (SP).

${ }^{7}$ Chefe do setor de Tumores Oculares Departamento de Oftalmologia da UNIFESP. São Paulo (SP).

Endereço para correspondência: Maria Cristina Martins - R. Joel Jorge de Melo, 236 - apto. 72 - São Paulo (SP) CEP 04128-080

E-mail: mcrism@uol.com.br

Recebido para publicação em 26.06.2003

Versão revisada recebida em 02.12.2004

Aprovação em 04.03.2005
}

\section{RESUMO}

Objetivo: Descrever e analisar os principais achados histopatológicos no retinoblastoma com ênfase na invasão de nervo óptico e túnicas oculares, correlacionando-os ao tratamento realizado. Métodos: Vinte e oito olhos com diagnóstico de retinoblastoma tratados por enucleação na Universidade Federal de São Paulo entre dezembro de 2000 e outubro de 2002 foram submetidos à revisão histopatológica. Os dados clínicos incluiram idade, sexo, raça, lateralidade e tratamento realizado. Na revisão histopatológica foram avaliados neovascularização da íris e seio camerular, grau de diferenciação do tumor e invasão do nervo óptico e coróide de acordo com a classificação de Khelfaoui. Resultados: De 27 pacientes, 13(48,1\%)eram do sexo masculinoe $14(51,9 \%)$ eram do sexo feminino, $16(59,3 \%)$ eram da raça branca, 5(18,5\%)eram daraça amarela, 4(14,8\%) erampardose 2(7,4\%)eram negros. A média da idade dos pacientes foi de 22,7 meses, sendo 13 casos bilaterais e 14 casos unilaterais. Com relação ao tratamento utilizado, 13 (46,4\%) olhos foram submetidos a enucleação primáriae 15 (53,6\%) tiveram tratamento prévio. Na avaliação histopatológica, $15(53,6 \%)$ tumores eram bem diferenciados. Neovascularização da íris e/ou seio camerular foram observados em $60,6 \%$ e necrose e calcificação em $89,2 \%$ dos casos. O envolvimento da coróide foi observado em 18 (64,2\%) dos casos (grau II e III) e a invasão de nervo óptico em 8 (28,5\%) (graus II, III e IV). Dos casos submetidos ao tratamento prévio $40 \%$ tinham algum grau de invasão das túnicas oculares e apenas $6,67 \%$ apresentavam simultaneamente invasão do nervo óptico e das túnicas oculares. Entretanto nos olhos que foram submetidos à enucleação primária 30,7\% apresentavam invasão das túnicas oculares e 53,8\% apresentavam simultaneamente invasão do nervo óptico e das túnicas oculares. Conclusão: Neovascularização, necrose ecalcificação foram achados mais comumente observados. A invasão do nervo óptico e coróide foram os dois fatores prognósticos mais importantes, encontrados em $28,5 \%$ e $64,2 \%$ dos casos respectivamente. Os casos tratados com enucleação primária apresentavam maior comprometimento ocular.

Descritores: Retinoblastoma/fisiopatologia; Neoplasias da coróide/patologia; Neoplasias do nervo óptico/patologia; Invasividade neoplásica; Fatores de risco

\section{INTRODUÇ̃̃O}

O retinoblastoma é o tumor intra-ocular mais freqüente na infância, em torno de 1 para 25.000 nascidos vivos ${ }^{(1-6)}$. Esse tumor representa de 2,5 a $4 \%$ de todos os tumores da infância ${ }^{(3)}$, não apresentando predileção sexual ${ }^{(4,6)}$.

O diagnóstico é geralmente feito antes dos 3 anos de idade ${ }^{(3-4,6)}$. Os casos bilaterais são diagnosticados nos dois primeiros anos de vida, ocorrendo em 20 a $35 \%$ dos casos. O quadro clínico depende do estágio da doença, tamanho e localização do tumor ${ }^{(3)}$.

A forma de crescimento da neoplasia pode ser: exofítica, endofítica, mista e 
difusa. O retinoblastoma é composto por células de origem neuroblástica com núcleo grande e basofílico, formato e tamanho variável e numerosas figuras de mitose podem ser observadas ${ }^{(3,6)}$.

A formação de rosetas descritas separadamente por Flexner e Wintersteiner, e de "floretes" podem ser consideradas formas de diferenciação das células tumorais em fotorreceptores ${ }^{(3,6)}$.

Um aspecto característico deste tumor é a sua capacidade de crescer mais rapidamente que o aporte sangüíneo disponível, sendo freqüente o achado de tufos de células viáveis com um vaso sanguíneo central ("pseudoroseta") e necrose e calcificação a partir de 90 a 110 micrômetros do lúmen vascu$\operatorname{lar}^{(3,6)}$. Essa calcificação distrófica é comumente identificada nos estudos de imagens (ultra-sonografia e tomografia computadorizada) para o diagnóstico do tumor.

A invasão do nervo óptico e das túnicas oculares são considerados importantes achados histopatológicos relacionados ao prognóstico ${ }^{(7)}$.

Khelfaoui et al, com base no exame histopatológico e evolução clínica de 172 pacientes, propuseram a divisão em 5 grupos para invasão de túnicas oculares e 4 para a invasão de nervo óptico (Quadro I). Segundo esses autores, a invasão de nervo óptico além da lâmina crivosa e/ou invasão maciça de coróide estariam associados a uma maior freqüência de desenvolvimento de doença disseminada após enucleação ${ }^{(8)}$.

O tratamento depende do estadiamento da lesão, sendo que a enucleação permanece como a forma mais comum de tratamento ${ }^{(4)}$. Entretanto os recentes avanços no entendimento do retinoblastoma tem proporcionado novas modalidades de tratamento como quimioterapia e tratamento local (placa radioativa, fotocoagulação a LASER, termoterapia transpupilar $\{\mathrm{TTT}\} \mathrm{e}$

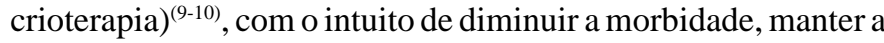
função visual e permitir a excelente sobrevida ${ }^{(11)}$.

\section{OBJETIVOS}

Descrever e analisar os principais achados histopatológicos no retinoblastoma com ênfase na invasão de nervo óptico e túnicas oculares correlacionando esses achados com o tratamento realizado.

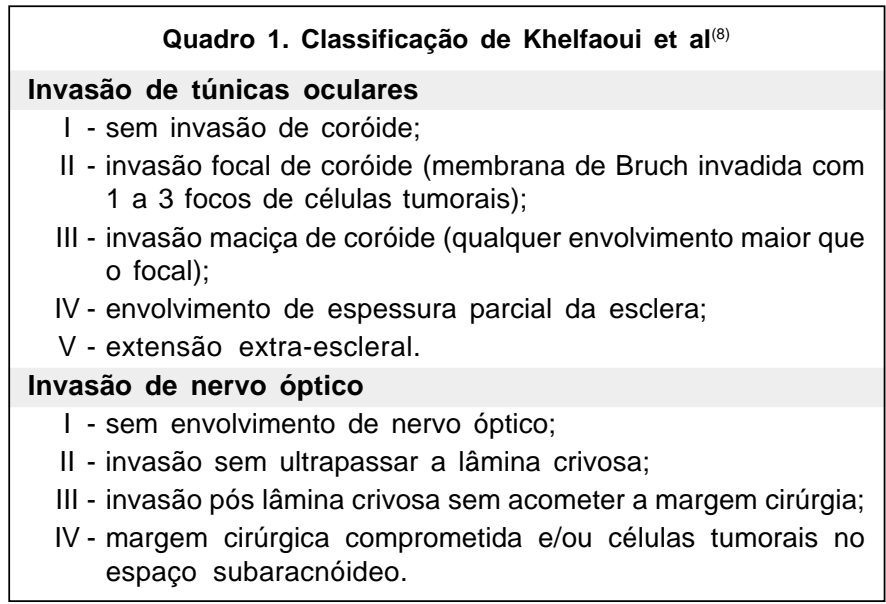

\section{MÉTODOS}

Foram estudados, após aprovação pelo Comitê de Ética da Universidade Federal de São Paulo, 28 olhos de 27 pacientes, consecutivamente, com diagnóstico de retinoblastoma que foram submetidos a enucleação entre dezembro de 2000 a outubro de 2002, no setor de tumores, do Departamento de Oftalmologia da Universidade Federal de São Paulo.

Foram avaliados a idade dos pacientes em meses no momento da enucleação, sexo, raça (branca, negra, amarela ou parda), a lateralidade (uni ou bilateral) e o tratamento (enucleação primária ou tratamento prévio a enucleação) instituído em todos os casos.

Os olhos foram fixados em formol a 10\%, por no mínimo 24 horas e, depois em álcool etílico a 70\%, por 24 horas e seccionados horizontalmente em um corte sagital no sentido pupilanervo óptico, passando pelo centro do tumor, quando possível. Foram realizadas a observação macroscópica com lupa esterioscópica (Leica MZ8) e documentação fotográfica do espécime.

Os espécimes (anel e coto distal do nervo óptico), foram processados separadamente para o estudo histopatológico, seguindo as técnicas rotineiras de inclusão em parafina. Foram realizados cortes de aproximadamente $5 \mathrm{~mm}$ e as lâminas coradas com hematoxilina-eosina $(\mathrm{HE})$ e ácido periódico de Schiff (PAS).

$\mathrm{Na}$ avaliação histopatológica realizado por um experiente patologista ocular (MCM) o grau de diferenciação foi classificado baseada na porcentagem de rosetas de Flexner-Wintersteiner presentes, sendo considerado um tumor bem diferenciado quando mais de $80 \%$ da área total do tumor apresentasse rosetas e pouco diferenciado quando estas fossem ausentes. $\mathrm{O}$ restante dos tumores foram classificados como moderadamente diferenciados ${ }^{(8,12)}$. O grau de invasão da coróide e esclera e o envolvimento do nervo óptico, foram classificados de acordo com a classificação de Khelfaoui et al ${ }^{(8)}$ (Quadro I). Foram observados ainda: neovascularização de íris e/ou seio camerular, necrose e calcificação.

\section{RESULTADOS}

Dos 28 olhos estudados de 27 pacientes, $13(48,1 \%)$ eram do sexo masculino e $14(51,9 \%)$ do feminino, $16(59,3 \%)$ eram da raça branca, $5(18,5 \%)$ eram da raça amarela, $4(14,8 \%)$ eram pardos e $2(7,4 \%)$ eram negros. A idade média dos pacientes no momento da enucleação era de 22,7 meses, variando de 2 meses a 96 meses.

O tumor era bilateral em $13(48,1 \%)$ casos e unilateral em 14 $(51,9 \%)$ casos. Com relação ao tratamento utilizado, $13(46,4 \%)$ olhos foram submetidos à enucleação primária e $15(53,6 \%)$ olhos tiveram tratamento prévio à enucleação. A quimioterapia isolada foi utilizada em 7 casos $(46,6 \%)$, quimioterapia associada com LASER (TTT) em 4 (26,6\%), quimioterapia associada a radioterapia externa em $2(13,4 \%)$, quimioterapia associada a LASER (TTT) e braquiterapia, em 1 paciente 
$(6,7 \%)$ e 1 caso $(6,7 \%)$ submetido inicialmente à vitrectomia seguido de quimioterapia. Dos 14 pacientes (15 olhos) que receberam tratamento prévio $10(71,4 \%)$ eram casos bilaterais e $3(20 \%)$ olhos evoluíram para atrofia bulbar.

$\mathrm{Na}$ avaliação histopatológica, o tumor apresentava-se caracterizado pela proliferação de pequenas células com alta relação núcleo/citoplasma, sendo bem diferenciado em 15 $(53,6 \%)$ olhos e moderamente diferenciado em $10(35,7 \%)$ olhos. Três olhos apresentavam-se com regressão do tumor sem células malignas viáveis. Necrose e calcificação foram observadas em $25(89.2 \%)$ e $17(60.7 \%)$ dos olhos apresentavam neovascularização de ângulo e/ou íris.

Quando analisado o grau de comprometimento do nervo óptico (Gráfico I) foram encontrados 20 olhos $(71,4 \%)$ com grau I (sem envolvimento de nervo óptico), 3 olhos $(10,8 \%)$ com grau II (invasão pré-laminar), 4 olhos (14,3\%) com grau III (invasão pós lâmina crivosa sem acometer a margem cirúrgica - Figura 1) e 1 olho (3,5\%) com grau IV (margem cirúrgica distal do nervo óptico comprometida).

Com relação ao comprometimento das túnicas oculares (Gráfico II) foram observados 7 olhos (25\%) com grau I (sem

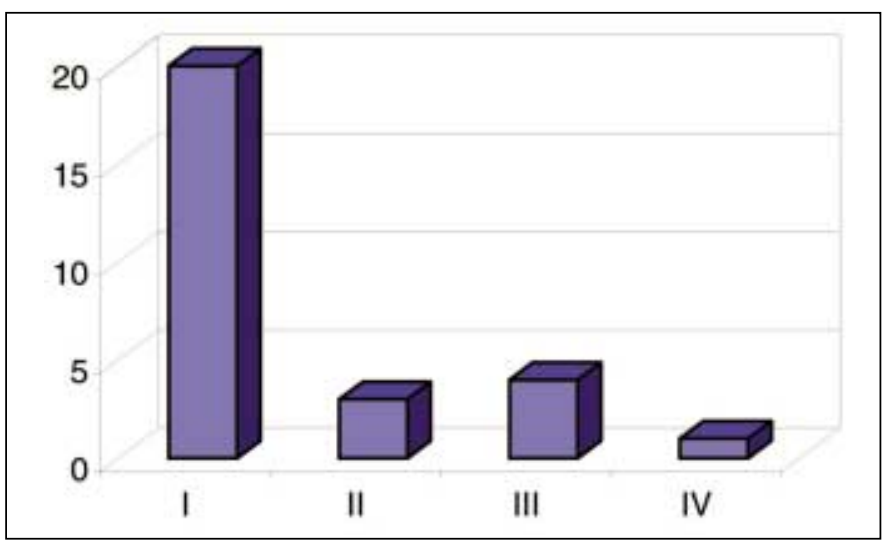

Gráfico I - Grau de invasão do nervo óptico de acordo com a classificação de Khelfaoui et al ${ }^{(8)}$

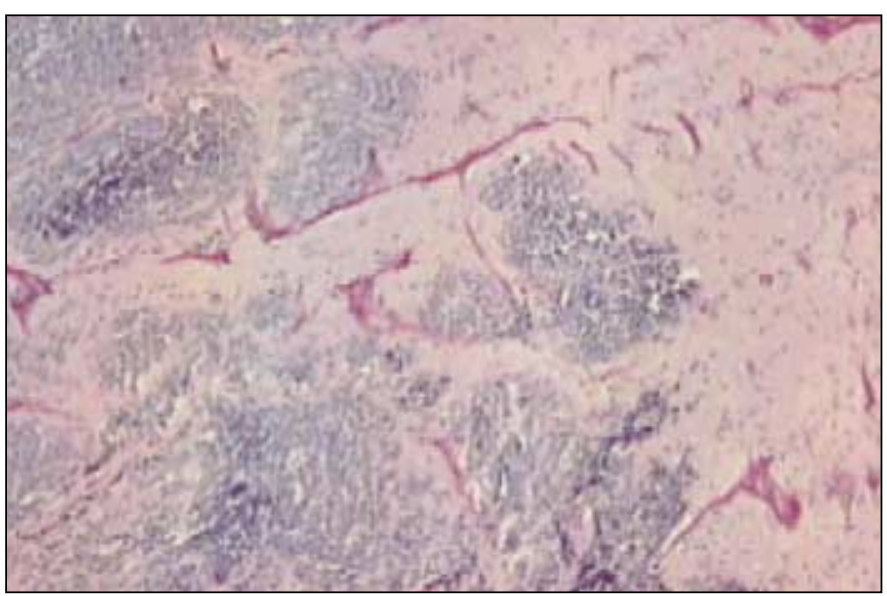

Figura 1 - Invasão do nervo óptico (H \& E, original magnificação 40 X)

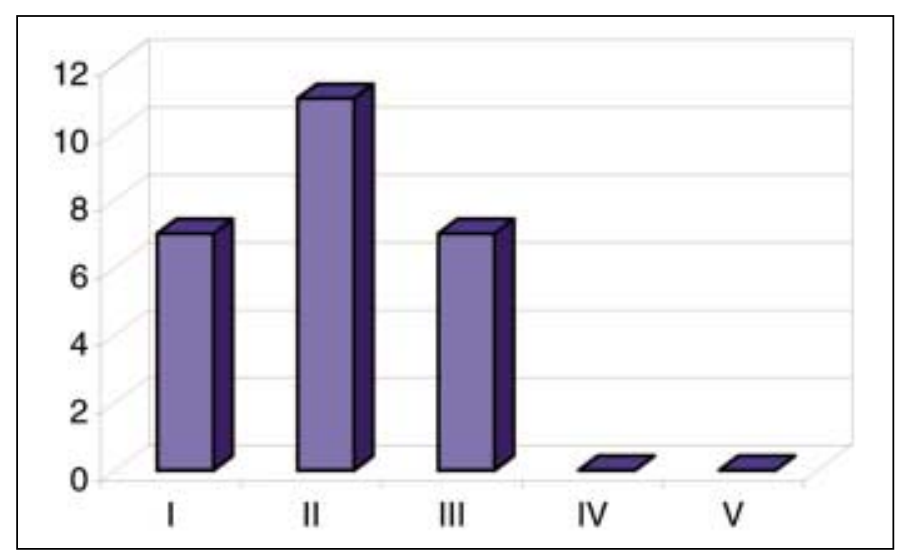

Gráfico II - Grau de invasão das túnicas oculares de acordo com a classificação de Khelfaoui et al ${ }^{(8)}$

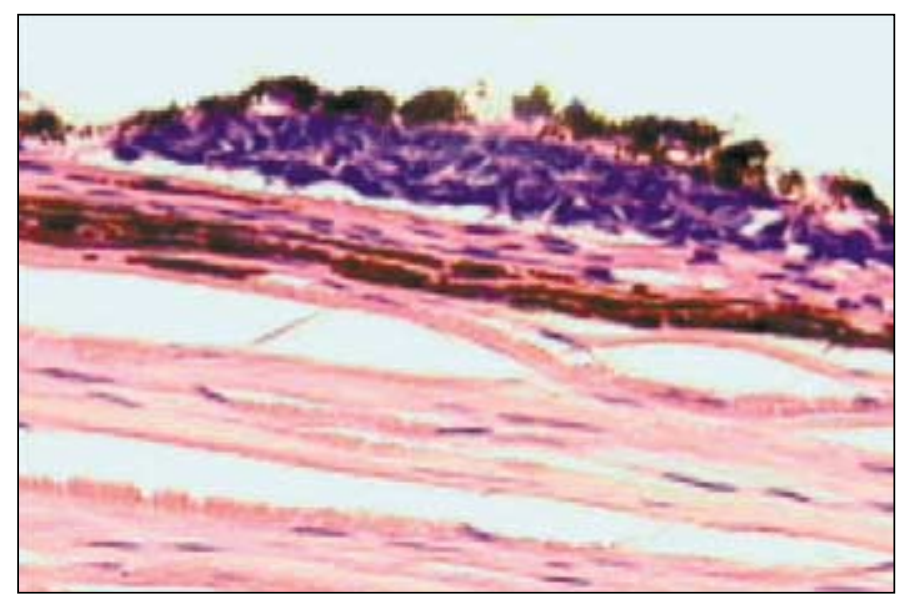

Figura 2 - Invasão focal de coróide (H \& E, original magnificação 200X)

invasão de coróide), 11 olhos (39,2\%) com grau II (invasão focal de coróide - Figura 2) e 7 olhos (25\%) com grau III (invasão maciça de coróide), em 3 olhos (10,8\%) não pode ser avaliado o grau de invasão pois os mesmos apresentavam atrofia bulbar.

Quando analisados separadamente os casos que foram submetidos à enucleação primária e os que receberam tratamento prévio, observou-se nos primeiros comprometimento do nervo óptico: grau I - 46,2\% (6), grau II - 15,3\% (2), grau III, $30,8 \%$ (4) e grau IV - 7,7\% (1). Quanto ao comprometimento das túnicas oculares $15,3 \%$ (2) eram grau I, 61,6\% (8) eram grau II e 23,1\% (3) eram grau III. Quando analisados os olhos que foram submetidos ao tratamento prévio, encontrou-se o comprometimento do nervo óptico: 93,3\% (14) eram grau I e 6,7\% (1) eram grau II. E na avaliação do comprometimento das túnicas oculares, observou-se que 33,3\% (5) eram grau I, $26,6 \%$ (4) eram grau II e 20\% (3) eram grau III (Tabela I). Observou-se que dos 15 olhos submetidos ao tratamento prévio nenhum tinha invasão isolada do nervo óptico, $6(40 \%)$ tinham algum grau de invasão das túnicas oculares e $1(6,67 \%)$ olho apresentava simultaneamente invasão do nervo óptico e 


\begin{tabular}{|c|c|c|c|}
\hline Tipo de tratamento & Comprometimento & Grau & n (\%) \\
\hline \multirow[t]{6}{*}{ Quimioterapia (7) } & Nervo óptico & I & $6(85,7)$ \\
\hline & & II & $1(14,3)$ \\
\hline & Túnicas oculares & I & $3(42,8)$ \\
\hline & & II & $2(28,6)$ \\
\hline & & III & $1(14,3)$ \\
\hline & & * & $1(14.3)$ \\
\hline \multirow[t]{3}{*}{ Quimioterapia + LASER (4) } & Nervo óptico & I & $4(100,0)$ \\
\hline & Túnicas oculares & I & $2(50,0)$ \\
\hline & & III & $2(50,0)$ \\
\hline \multirow[t]{2}{*}{ Quimioterapia + Radioterapia (2) } & Nervo óptico & I & $2(100,0)$ \\
\hline & Túnicas oculares & * & $2(100,0)$ \\
\hline \multirow[t]{2}{*}{ Quimioterapia + LASER + Braquiterapia (1) } & Nervo óptico & 1 & $1(100,0)$ \\
\hline & Túnicas oculares & II & $1(100,0)$ \\
\hline \multirow[t]{2}{*}{ Vitrectomia e Quimioterapia $(1)^{\star *}$} & Nervo óptico & 1 & $1(100,0)$ \\
\hline & Túnicas oculares & II & $1(100,0)$ \\
\hline
\end{tabular}

das túnicas oculares. Nos 13 olhos que foram submetidos à enucleação primária nenhum tinha invasão isolada do nervo óptico, $4(30,7 \%)$ apresentavam invasão das túnicas oculares e $7(53,8 \%)$ apresentavam simultaneamente invasão do nervo óptico e das túnicas oculares.

\section{DISCUSSÃO}

A freqüência de invasão do nervo óptico em olhos enucleados por retinoblastoma varia de acordo com a literatura de $25 \%$ a $63,6 \%{ }^{(3,13)}$. A invasão do nervo óptico até o plano de secção cirúrgica é considerada como fator de mau prognóstico. Nesses pacientes, a mortalidade varia entre $50 \%$ a $81 \%(3,13)$.

A utilização da quimioterapia e modalidades de tratamento local, está associada a uma diminuição da morbidade ${ }^{(11)}$. No presente estudo foram incluídos 14 pacientes $(51,8 \%)$ submetidos à quimioterapia (quimiorredução) prévia, isolada ou associada a tratamento local, seguido de enucleação. Um dos pacientes, foi submetido à inadvertida vitrectomia em outro serviço, sendo tratado com quimioterapia seguido de enucleação. Um outro paciente com doença bilateral, foi submetido ao tratamento prévio e teve ambos os olhos enucleados.

A enucleação, na maioria dos casos onde o estadiamento determina apenas comprometimento intra-ocular é curativa. Em alguns casos, entretanto, há evolução para doença sistêmica mesmo após esse procedimento cirúrgico radical. A ocorrência de disseminação da doença se associa com queda significativa da sobrevida ${ }^{(14)}$.

A invasão pós-laminar do nervo óptico, mesmo com margem cirúrgica livre (coto distal), é considerada pela maioria dos autores como fator de risco para a disseminação da doença. Khelfaoui et al, encontraram doença extra-ocular em $32 \%$ dos casos com invasão pós-laminar e margem livre ${ }^{(8)}$. Outros autores relatam nestes casos, mortalidade variando de 13 a $69 \%{ }^{(13)}$. No presente estudo foram encontrados 8 olhos
(28,5\%) com invasão do nervo óptico, sendo 3 olhos com invasão pré-laminar, 4 olhos com invasão laminar e 1 olho com margem cirúrgica comprometida.

A invasão de coróide como fator de risco para disseminação da doença é ainda questionada ${ }^{(3,13)}$. Alguns autores sugerem que o envolvimento da coróide é um fator de risco significante para a disseminação extra-ocular, com mortalidade de 11 a $81 \%{ }^{(13)}$. Alguns autores consideram a invasão de coróide um fator aditivo, que só implica em maior risco de doença disseminada, quando associado a outros fatores de risco, como a invasão pós-laminar do nervo óptico ${ }^{(15)}$.

A invasão da coróide foi observada em diferentes estudos variando de 12 a $62 \%$ dos olhos enucleados por retinoblasto$\mathrm{ma}^{(3)}$. Em um estudo de 230 casos de retinoblastoma foram observados o comprometimento da coróide em $82 \%$ dos olhos, porém não foi encontrado nestes casos uma relação direta entre o comprometimento da coróide e metástases ${ }^{(16)}$. Foram observados no presente estudo $64,2 \%$ dos olhos com envolvimento da coróide.

Ainda observamos que $10(35,7 \%)$ olhos apresentavam algum grau de invasão das túnicas oculares, e em 8 (28.5\%) olhos notou-se invasão simultânea das túnicas oculares e do nervo óptico.

Quando se comparou o grau de envolvimento ocular entre os casos que foram submetidos à enucleação primária com os casos submetidos ao tratamento prévio, observou-se que 7 casos $(53,8 \%)$ que sofreram enucleação primária tiveram comprometimento do nervo óptico e que 11 casos $(84,6 \%)$ tiveram comprometimento das túnicas oculares. Já nos casos que tiveram tratamento prévio apenas 1 caso $(6,6 \%)$ apresentava comprometimento do nervo óptico e 7 casos $(46,6 \%)$ apresentavam comprometimento das túnicas oculares.

Isso provavelmente se deve ao fato de que os olhos submetidos à enucleação primária apresentavam doença clinicamente avançada e que este foi o tratamento de escolha. Entretanto, os olhos submetidos ao tratamento prévio à apresentação inicial, 
oferecendo a possibilidade de tentativa de um tratamento conservador, tiveram na sua evolução, uma resposta insatisfatória e foram submetidos à enucleação.

Foram encontrados 17 olhos $(60,7 \%)$ com neovascularização da íris e/ou ângulo. A neovascularização da íris já foi descrita previamente em olhos enucleados por retinoblastoma, sendo descritos em 31 a $80 \%$ dos espécimes examina$\operatorname{dos}^{(17-18)}$, sendo descrito sua associação com fatores histológicos de estágios avançados da doença (invasão de coróide e nervo óptico).

Quando comparamos os olhos que tiveram enucleação primária observamos que $11(84,6 \%)$ apresentavam neovascularização de íris e/ou ângulo, enquanto que nos olhos que tiveram tratamento prévio apenas $6(40 \%)$ apresentavam essa patologia.

Em um estudo sobre achados histopatológicos em retinoblastoma tratados com quimioterapia foram descritos dois casos de regressão tumoral, com substituição do tumor por tecido glial ${ }^{(19)}$. No presente estudo observamos três olhos, um tratado com quimioterapia isolada e dois tratados com quimioterapia e radioterapia, com regressão tumoral.

Os resultados aqui apresentados são um estudo preliminar dentro de uma série de casos enucleados seqüencialmente no setor de tumores oculares da UNIFESP - EPM, onde se tentará estabelecer a relação entre os achados histopatológicos e a evolução dos pacientes ao longo dos anos.

\section{CONCLUSÕES}

1 - Nos olhos enucleados, neovascularização de íris e ângulo, necrose e calcificação foram os achados histopatológicos mais comumente observados nos casos de retinoblastoma.

2 - A invasão do nervo óptico e coróide, considerados dois importantes fatores de prognóstico foram observados em $28,5 \%$ e $64,2 \%$ dos olhos respectivamente.

3 - Os casos tratados com enucleação primária apresentavam maior comprometimento do nervo óptico e das túnicas oculares quando comparados aos olhos submetidos ao tratamento prévio.

\section{ABSTRACT}

Objetive: To study histopathological findings of enucleated eyes with retinoblastoma. Methods: Twenty-eight cases of retinoblastoma treated by enucleation at the Federal University of São Paulo from December 2000 to October 2002 were histopathologically reviewed. Clinical data included age, gender, race, unilateral or bilateral involvement and previous treatment. The histopathological review evaluated the presence of iris and/or angle neovascularization, tumor differentiation and optic nerve and choroidal invasion according to Khelfaoui's classification. Results: Of 27 patients, 13 (48.5\%) were boys and $14(59.3 \%)$ were girls, 16 were white, 6 were black and 5 were asiatic, age ranging from 2 to 96 months (mean, 22.7 months). 13 cases were bilateral and 14 cases were unilateral. All tumors were histologically characterized by a proliferation of small cells with high nuclear-to-cytoplasmic ratios and $20(71.4 \%)$ of them were well differentiated. Choroidal involvement was observed in 18 $(64.2 \%)$ cases (degree II, III) and optic nerve invasion in 8 (28.5\%) cases (degree III, IV, V). Conclusion: Neovascularization, necrosis and calcification were the most commonly observed feature. The invasion into the optic nerve and choroid, which are the two most important predictors of patient outcome were found in $28.5 \%$ and $64.2 \%$ of the cases, respectively.

Keywords: Retinoblastoma/physiopathology; Choroid neoplasms/pathology; Optic nerve neoplasms/pathology; Neoplasms invasiveness; Risk factors

\section{REFERÊNCIAS}

1. Lira RP, Leôncio MP, Pinho P, Rocha G, Lira PC. Retinoblastoma extra-ocular: estudo de 37 casos. Arq Bras Oftalmol. 1995;58(6):480-3.

2. Kronbauer FL, Corrêa ZM, Tyllmann C, Escovar CE, Marcon IM. O uso da quimioterapia no tratamento do retinoblastoma: avaliação retrospectiva. Arq Bras Oftalmol. 2000;63(6):481-6.

3. Marback, ED. Angiogênese tumoral como fator tumoral para disseminação da doença em retinoblastoma [tese]. São Paulo: Universidade Federal de São Pau$10 ; 2001$.

4. Kamski JJ, Singh A. Tumors of the retina. In: Kamski JJ. Clinical ophthalmology: a systematic approach. $3^{\text {rd }}$ ed. Oxford: Butterrworth-Heinemann; 1994. p.222-6.

5. Nehemy MB, Nehemy AL, Silva FA, Miranda D. Retinoblastoma em um olho atrófico. Arq Bras Oftalmol. 1991;54(2):90-2.

6. McLean IW. Retinoblastomas, retinocytoma, end pseudoretinoblastoma. In: Spencer WH, ed. Ophthalmic pathology: an atlas and textbook. $4^{\text {th }}$ Philadelphia: WB Saunders; 1996. p.1340-75.

7. Singh AD, Shields CL, Shields JA. Prognostic factors in retinoblastoma. J Pediatr Ophthalmol Strabismus. 2000;37(3):134-41.

8. Khelfaoui F, Validire P, Auperin A, Quintana E, Michon J, Pacquement H, et al. Histopathologic risk factors in retinoblastoma. A retrospective study of 172 patients treated in a single institution. Cancer. 1996;77(6):1206-13.

9. Shields CL, Santos MC, Diniz W, Gündüz K, Mercado G, Cater JR, et al. Thermotherapy for retinoblastoma. Arch Ophthalmol. 1999;117(7):885-93.

10. Lumbroso L, Doz F, Urbieta M, Levy C, Bours D, Asselain B, et al. Chemothermotherapy in the management of retinoblastoma. Ophthalmology. 2002; 109(6):1130-6.

11. Friedman DL, Himelstein B, Shields CL, Shields JA, Needle M, Miller D, et al. Chemoreduction and local ophthalmic therapy for intraocular retinoblastoma. J Clin Oncol. 2000;18(1):12-7.

12. Schouten-van Meeteren AY, Van der Valk P, Van der Linden HC, Moll AC, Imhof SM, Huismans DR, et al. Histopathologic features of retinoblastoma and its relation with in vitro drug resistance measured by means of the MTT assay. Cancer. 2001;92(11):2933-40.

13. Uusitalo MS, Van Quill K, Scott IU, Matthay KK, Murria TG, O’Brien JM. Evaluation of chemoprophylaxis in patients with unilateral retinoblastoma with high-risk features on histopathologic examination. Arch Ophthalmol 2001; 119(1):41-8.

14. Rubin CM, Robison LL, Cameron JD, Woods WG, Nesbit ME, Krivit W, et al. Intraocular retinoblastoma group $\mathrm{V}$ : an analysis of prognostic factors. J Clin Oncol. 1985;3(5):680-5.

15. Magramm I, Abramson DH, Ellsworth RM. Optic nerve involvement in retinoblastoma. Ophthalmology. 1989;96(2):217-22.

16. Pérez Samatier C, Travezán Carvo R, Salem Abugattas LE. Retinoblastoma: Histopathology study of 230 cases. Acta Cancerol. 1989;20(1):5-10.

17. Pe'er J, Neufeld M, Baras M, Gnessin H, Itin A, Keshet E. Rubeosis iridis in retinoblastoma. Histologic findings and the possible role for vascular endothelial growth factor in its induction. Ophthalmology. 1997;104(8):1251-8.

18. Shields CL, Shields JA, Baez K, Cater JR, De Potter P. Optic nerve invasion of retinoblastoma. Metastatic potential and clinical risk factors. Cancer. 1994;73(3): 692-8.

19. Demirci H, Eagle RC Jr, Shields CL, Shields JA. Histopathologic findings in eyes with retinoblastoma treated only with chemoreduction. Arch Ophthalmol. 2003;121(8):1125-31. 